\title{
Galaxy Harassment and the Evolution of Clusters of Galaxies
}

\author{
Ben Moore ${ }^{1}$, Neal Katz ${ }^{1}$, George Lake円, \\ Alan Dressler 2 and Augustus Oemler, Jr?
}

\begin{abstract}
Disturbed spiral galaxies with high rates of star formation pervaded clusters of galaxies just a few billion years ago, but nearby clusters exclude spirals in favor of ellipticals. "Galaxy harassment" (frequent high speed galaxy encounters) drives the morphological transformation of galaxies in clusters, provides fuel for quasars in subluminous hosts and leaves detectable debris arcs. Simulated images of harassed galaxies are strikingly similar to the distorted spirals in clusters at $z \sim 0.4$ observed by the Hubble Space Telescope.
\end{abstract}

Clusters of galaxies are unique cosmological laboratories. There are several hundred galaxies moving at relative velocities up to several thousand $\mathrm{km} \mathrm{s}^{-1}$ in regions no larger than the distance between the Milky Way and its nearest neighbor, the Andromeda galaxy (M31). Clusters of galaxies have been observed at redshifts up to $2^{1}$. By understanding their evolution over cosmic times, we probe the geometry of the Universe and the development of its largest structures.

Nearby rich galaxy clusters are dominated by elliptical "E" and lenticular "S0" galaxies" mostly low luminosity dwarfs. Twenty years ago Butcher and Oemler ${ }^{3,4}$ discovered that clusters at $z \gtrsim 0.4$ have a substantial population of "blue galaxies" seen only as fuzzy blobs in their ground based images. Recent Hubble Space Telescope (HST) images revealed that the "fuzzy blue blobs" are low luminosity, often disturbed, spiral galaxies "Sp" $5-8$. The HST imaging teams stress that the disturbed blue galaxies are ubiquitous, but few have other galaxies nearby ${ }^{5}$ and there were multiple bursts of star formation spanning up to $2 \mathrm{Gyr}^{8}$. The dramatic transformation of clusters (shown in Figure 1) occured during a "look-back time" of just 4-5 billion years, only a few cluster orbital times. In contrast, the morphological fraction in the field shows far less evolution ${ }^{9}$.

Figure 1: The dramatic evolution of clusters from $z \sim 0.4$ to today is shown in these images of their inner $300 \mathrm{~h}^{-1} \mathrm{kpc}$, where $h$ is the Hubble constant in units of $100 \mathrm{~km} \mathrm{~s}$ $M_{p c^{-1}}$. The left panel is the nearby Coma cluster at $z=0.023$ (courtesy A. Fruchter, B. Moore and C. Steidel). Nearly every object surrounding the two central dominant elliptical galaxies is an E or SO. The right panel is an HST image of CL0939 at $z=0.41^{6}$. This cluster is dominated by spiral galaxies; many appear disturbed yet no other galaxies are nearby. While others appear normal, they have enhanced rates of star formation betrayed by strong $\mathrm{H}_{\beta}$ and $\mathrm{OII}$ emission lines. The bright elliptical population is already in place at this redshift. The difference in galaxy populations is most dramatic at luminosities more than two magnitudes fainter than $L_{*}$, the characteristic break in the luminosity function ${ }^{10}$. Fainter than this luminosity, 90\% of galaxies in clusters at $z \sim 0.4$ are bulgeless "Sd" disk

\footnotetext{
${ }^{1}$ Department of Astronomy, University of Washington, Seattle, WA 98195-1580, USA

${ }^{2}$ Observatories of the Carnegie Institution of Washington, Pasadena, CA 91101-1292, USA

${ }^{3}$ Yale University Astronomy Department, New Haven, Ct 06511, USA
} 
systems, whereas $90 \%$ of galaxies within nearby clusters are dwarf ellipticals "dE" or dE/SO 11,12 . The resolution of both images is $\sim 0.5 h^{-1} \mathrm{kpc}$.

Given a mechanism for distorting galaxies and promoting star formation that operates when a spiral first enters a cluster, hierarchical clustering models will naturally enhance the number of "Butcher-Oemler clusters" at $z \sim 0.4^{13}$. Proposed mechanisms include: mergers ${ }^{14,15}$, compression of gas in the high pressure cluster environment ${ }^{16,17}$ and tidal compression by the cluster ${ }^{18,19}$. Each of these scenarios can produce star-bursts, but none address morphological evolution or identify the remnants of these distorted blue galaxies in present day clusters. By analyzing their HST images, Oemler et al ${ }^{20}$ conclude that merging is implausible as the blue galaxy fraction is large and the merging probability is low. They observe disturbed spirals throughout the cluster, whereas both ram pressure stripping and global tides will only operate efficiently near the cluster's center.

What mechanism drives star-bursts and rapid morphological evolution throughout a cluster of galaxies? Although direct mergers are extremely rare, every galaxy experiences a high speed close encounter with a bright galaxy once per Gyr. Here, "close" means within 50 kiloparsecs (several optical radii) and "bright galaxy" is one as luminous as $L_{*}$, the characteristic break in the galaxy luminosity function ${ }^{10}$. (The Milky Way has a luminosity of approximately $L_{*}$.) We use high resolution numerical simulations of galaxies in clusters to examine the resulting damage of these close encounters. To distinguish this from other collisional effects such as galaxy mergers and galaxy cannibalism, we refer to these frequent encounters as "galaxy harassment".

\section{The cluster environment and galaxy harassment}

We simulate the evolution of a small bulgeless spiral galaxy orbiting within a dense cluster modeled on Coma (Figure 1). Figure 2 shows an edge on view of the model galaxy along with an inventory of its stars, gas and dark matter. Some of our simulations use smoothed particle hydrodynamics ${ }^{21}$, to evolve the gas component of the disk at resolutions of 100 $500 \mathrm{pc}$.

We simulated galaxies on circular and elliptical orbits in smooth cluster potentials before examining the effects of harassment. The disk galaxy shows little evolution over 5 Gyrs when placed on a $450 \mathrm{kpc}$ circular orbit in a smooth cluster potential. The disk becomes bar unstable after the first pericentric passage ${ }^{18}$ when placed on an eccentric orbit with apocenter and pericenter at $600 \mathrm{kpc}$ and $300 \mathrm{kpc}$ respectively. Thereafter, each time the galaxy passes through pericenter, the halo loses a small fraction of its mass but stars and gas remain bound. The evolution is far more dramatic after including the other cluster galaxies.

Figure 2: An edge on view of a model Sd galaxy. Blue, red and yellow particles make up the dark matter, gas and stars respectively. The exponential disk has a scale length of 2.5 kpc and scale height of 200 pc. The disk is constructed with a Toomre "stability" parameter $Q=1.5$ and is run in isolation for 2 Gyr (as shown) before being set into orbit in the cluster. The gaseous disk is initially on cold circular orbits. The dark halo is a spherical isothermal with a core radius of one kpc that is tidally truncated at the pericenter of the galaxy's orbit. Within $20 \mathrm{kpc}$ the ratio of dark matter to stars to gas is 20:5:1 and the total mass is $\sim 10^{11} M_{\odot}$. The graph displays the contribution of each component to the rotational 
velocity of the disk. Using the relationship found by Tully \& Fisher ${ }^{22}$, a circular velocity of $\sim 160 \mathrm{~km} \mathrm{~s}^{-1}$ corresponds to a galaxy with a luminosity about $L_{*} / 5$. We performed similar simulations using model galaxies with a peak circular velocity of $\sim 110 \mathrm{~km} \mathrm{~s}^{-1}$. The galaxies are placed in a model of the Coma cluster that has a total mass within its virialised radius of $7 \times 10^{14} h^{-1} M_{\odot}$. Galaxies within the cluster are drawn from a Schechter luminosity function normalised to a typical cluster mass-to-light ratio of $250 \mathrm{~h}$. This results in a cluster with 950 galaxies brighter than the Magellanic clouds, but only 31 brighter than $L_{*}$. Internal velocity dispersions are assigned using the observed correlation with luminosity ${ }^{23}$. The assigned masses assume that the dark halos are tidally truncated at their pericentric distance reduced by a time averaged loss of $25 \%$ owing to harassment ${ }^{24}$. The fraction of the cluster's density attached to galaxies varies from zero at its center to nearly unity at its virial radius of 1.5 $h^{-1}$ Mpc. At $300 h^{-1} k p c$, roughly $15 \%$ of the mass is bound to galaxies. The rest of the cluster mass is in a smoothly distributed background represented by a fixed analytic potential. Further details can be found in Moore et al ${ }^{24}$.

When we include harassment, the havoc wreaked is determined by the masses of bright galaxies in clusters. Galaxies in the field have massive dark halos, but there has been speculation that these were stripped from individual galaxies within clusters ${ }^{25}$. All galaxies are tidally limited by the potential field of the cluster. Over the $5 \mathrm{Gyr}$ evolution of the cluster, bright galaxies retain more than half of the mass within their tidal radius (measured at the pericenter of their orbit), the rest being liberated by fast encounters with other bright galaxies $^{24}$.

We have been conservative in our simulations to insure that our results are robust. At a fixed mean orbital radius or "guiding center", the effects of harassment becomes stronger as orbits become more elongated. In our cluster model, the mean ratio of a perturbing galaxy's apocenter to its pericenter is greater than 10-to-1. A galaxy in a rich cluster with a guiding center of $450 \mathrm{kpc}$ will have a typical pericenter $\left(r_{\text {peri }}\right)$ slightly greater than 150 kpc. The masses we assign galaxies are $\sim 2.8 \times 10^{11}\left(r_{p e r i} / 150 k p c\right)\left(L / L_{*}\right)^{3 / 4} M_{\odot}$. As a result, their mass-to-light ratios are $M / L=44 h^{2}\left(r_{\text {peri }} / 100 k p c\right)\left(L / L_{*}\right)^{-1 / 4}$. The luminous parts of elliptical galaxies are observed to have mass-to-light ratios of $12 h M_{\odot} / L_{\odot}{ }^{26}$, so the perturbing galaxies have modest amounts of dark matter. However, we follow the evolution of individual harassed galaxies that have apo/peri ratios of 2 (i.e. apocenter at $600 \mathrm{kpc}$, pericenter at $300 \mathrm{kpc}$ ). As a result, this galaxy avoids extremes of the cluster distribution and starts with a large dark halo mass since its pericentric distance is not far from its guiding center. Both effects serve to underestimate the effects of harassment.

\section{The changing morphologies of harassed galaxies}

In Figure 3, we follow the evolution of a harassed galaxy through its slightly eccentric orbit. At each phase, we compare with images of galaxies in clusters. Our initial conditions look like a normal spiral galaxy found in the field (Figure 3a). Typically, the first encounters create "disturbed barred spirals" with sharp and dramatic features drawn out from the dynamically cold disk (Figure $3 \mathrm{~b}$ ). Tails of material can be pulled out and distorted by the tidal field of the cluster (Figure 3c). The gas distribution often forms ring structures that tumble within the stellar bar (Figure $3 \mathrm{~d}$ ). 
Figure 3: Comparisons of synthetic images from our simulations with observations of harassed galaxies. In the simulated images, the stellar distribution is smoothed and filtered to model the resolution of the observations. The limiting brightness contours are about 27 magnitudes per square arcsecond. (a) The left panel shows the initial model galaxy viewed face and edge on. The size of each image is $40 \mathrm{kpc}$ across. (b) The upper image shows a disturbed spiral galaxy taken from the Butcher-Oemler cluster CL144\%. The lower image shows our model galaxy 150 million years after suffering a single strong encounter that has pulled the two tails of material from the disk. At this time, the perturbing galaxy has already moved over $200 \mathrm{kpc}$ away. (c) The upper image is NGC 4438, a spiral galaxy near the center of the Virgo cluster (from Sandage and Bedke ${ }^{27}$ ). The close companion in this image is probably not responsible for the disturbance ${ }^{28}$. The lower image shows a snapshot of our model galaxy after a Gyr in the cluster. The tidal tails of material pulled from the galaxy have been subsequently tidally distorted. (d) The upper image is a spiral galaxy with a prominent ring in the distant rich cluster CL0939. The lower image shows the ring structure often observed in harassed galaxies. (e) Our model galaxy after 3 Gyrs of evolution. The stellar distribution shown here should be compared with the initial model shown in Figure $3 a$ and with the dwarf elliptical galaxies shown in Figure 3f. (f) CCD images of dwarf elliptical galaxies in the Coma cluster. The pixel scale is roughly the same physical size as shown in Figures 3(a-e).

The evolution is driven by several close encounters that would drive the multiple starbursts inferred from HST data ${ }^{8}$. Another observational puzzle has been the ubiquity of disturbed galaxies with no sign of current interaction ${ }^{6}$. None of the images of our model galaxy has another cluster galaxy within $50 \mathrm{kpc}$. Over the course of $3 \mathrm{Gyr}$, the closest approach of another galaxy is more than $30 \mathrm{kpc}$ away. Since the relative velocity of strong encounters is $\sim 1,500 \mathrm{~km} \mathrm{~s}^{-1}$, and the velocity impulse internal to the galaxy is only $\lesssim 50$ $\mathrm{km} \mathrm{s}^{-1}$, the perturbing galaxy moves $\sim 100 \mathrm{kpc}$ by the time the disk's response is visible.

After several strong encounters, the loss of angular momentum to their own dark halos and the perturbing galaxies, combined with impulsive heating, leads to a prolate figure supported equally by random motions and rotation. The gas sinks to the very center of the galaxy and the stellar distribution is heated to the extent that it closely resembles a dwarf elliptical, although some retain very thick stellar disks and would be classed as dwarf lenticulars. At this stage in the evolution encounters cease to create sharp distortions and fail to remove any more material from the compact remnant.

The final stellar systems have a large degree of rotational support, surface density profiles and shapes that are in good agreement with observations. Figure 3e shows the stellar configuration after 3 Gyrs and can be compared with real images of dwarf elliptical galaxies taken from the Coma cluster (Figure 3f). Note that the final photometric axes of the model galaxy are tilted with respect to the initial plane of rotation of the disk.

Using our simulations, we can identify the present-day remnants of the disturbed spirals seen at $z \sim 0.4$. Below $L_{*}$, two distinct classes of elliptical galaxies are observed. Low luminosity Es with high central surface brightness are a rare extension to the sequence of bright ellipticals; the archetype is M32. The most numerous galaxies in clusters are in a second class of dwarf ellipticals, also known as dwarf spheroidals (dE/dSph). Their exponential surface brightness profiles resemble those of spirals, as does the correlation of their low central surface brightnesses with total luminosity. They are faint, at least 3 magnitudes below $L_{*}$ and as many as 14 magnitudes if one extrapolates to the faintest known galaxies in the Local Group, Draco and Ursa Minor ${ }^{29,30}$. Harassment transforms 
spirals into this latter class of galaxies.

The observed stellar populations of $\mathrm{dE}$ galaxies implies recent star formation activity that can easily be understood in our model as a result of recent encounters with cluster galaxies. Harassed Sd spiral galaxies undergo a remarkable transformation from one morphological class to another without any merging taking place. Their dynamical states can account for all of the dissimilarities between dwarf elliptical and normal elliptical galaxies. Harassment provides the link between the dominant populations of galaxies in clusters at $z \sim 0.4$ and the present-day.

\section{Other effects of harassment: tidal debris and quasar fuel}

Stellar and gaseous material torn from the disk during violent encounters creates debris tails that lead and follow the galaxy's orbit through the cluster. At pericenter, the tidal debris creates a giant arc that could be mistaken for a gravitational arc when viewed faceon (Figure 4). However, the transverse size is slightly greater than a lensed galaxy and its redshift will match the cluster. The harassment of the debris tails should create tidal shocks that promote the formation of dwarf galaxies, as seen in observations and simulations of tails in merging galaxies ${ }^{31,32}$. Low surface brightness features punctuated with dwarf galaxy formation should be detectable in most HST images of $z \sim 0.4$ clusters.

Figure 4: The smoothed surface brightness of the stellar tidal debris after 4 Gyrs of evolution. The image is 2 Mpc across and the intensity of the colour shows the logarithm of the smoothed stellar surface density plotted wherever the surface brightness $m_{b}<30$ magnitudes per square arcsecond. The white dots are individual particles from the galaxy's dark halo. The two long tails were stripped by strong encounters with other cluster members coupled with the mean tidal field of the cluster. At various positions along the orbital path the stripped stars arrange themselves into long arcs following the galaxy's orbit. HST images should show the brightest features (where the colour is brighter than orange).

Future observations of intra-cluster light coupled with simulations covering the large parameter space of orbits and luminosities will provide interesting constraints on our evolutionary scenario. Current observations are conflicting, anywhere from $0 \%$ to $30 \%$ of the total cluster luminosity could reside in a diffuse component ${ }^{33,34}$. In our cluster model, $20 \%$ of the light starts off in galaxies fainter than $L_{*} / 5$. After 5 Gyrs, $\sim 20 \%$ of the stars are lost to the intra-cluster medium from our small Sd galaxies. The bulk of the evolution is driven by the few $(\lesssim 5)$ strong encounters with galaxies brighter than $\sim L_{*}$. As a result, the evolution is chaotic: whereas one fragile disk galaxy can avoid strong encounters for a few Gyrs, another may be completely destroyed. The total quantity of stripped material can vary from a few percent, to rarer cases where the entire galaxy is disrupted after several strong encounters.

Recent HST images of quasars suggest that many quasar hosts are not galaxies as luminous as $L_{*}{ }^{36}$. This is surprising as simple energy considerations imply that quasars need $10^{8} M_{\odot}-10^{9} M_{\odot}$ to fuel the black hole engine. To be conservative, one would prefer models where no more than $10 \%$ of a galaxy's gas must be channeled to the center. This argues for hosts at least as large as our own Milky Way, which has a few times $10^{9} M_{\odot}$ of gas. From 8 HST images of low redshifts quasars, Bahcall et al ${ }^{36}$ found that 5 of the host galaxies must be 0.5 to 1.5 magnitudes fainter than $L_{*}$. Spiral galaxies of this luminosity 
have gas masses of order $10^{8} M_{\odot}$, a quasar needs nearly all of it for fuel!

Galaxy harassment can give the quasar the fuel it needs. While $10 \%$ of the gas is tidally stripped from the galaxy, the remaining $90 \%$ of the gas $\left(\gtrsim 10^{8} M_{\odot}\right)$ sinks to the inner few hundred parsecs - the resolution of our simulation - by rapidly losing angular momentum to the perturbing galaxies, dark halo and stellar bar.

Clearly, many quasars have luminous hosts ${ }^{36}$. Also, quasars are known to avoid rich clusters $^{37}$, so how could they be harassed? Oddly, three of the five quasars with subluminous

hosts found by Bahcall et al ${ }^{35}$ lie in clusters. A fourth is probably in a cluster and the environment of the fifth has not been studied. Harassed galaxies provide ideal hosts for quasars at intermediate redshifts known to lie in subluminous galaxies ${ }^{38}$.

\section{Concluding Remarks}

Galaxies are metamorphised by their mutual interactions. "Merging" of spirals in groups creates bright ellipticals" ${ }^{39}$. In a cluster, one of these "cannablises" its neighbors to become the giant central ellpitical ${ }^{40}$. The dwarf ellipticals are created by the harassment of low luminosity spirals. Harassment has the potential to change any internal property of a galaxy within a cluster including the gas distribution and content, the orbital distribution of stars and the overall shape. Our first examination has only touched on some of the most dramatic changes, the phenomenology of harassment promises to be even richer than that of merging and cannibalism.

Acknowledgments This research was funded by NASA through the LTSA, ATP and HPCC/ESS programs. 


\section{References}

1. Dressler A, Oemler A., Gunn J.E. \& Butcher H. 1993, Astrophys.J. Lett., 404, L4-6.

2. Dressler A. 1980, Astrophys.J., 236, 351-65.

3. Butcher H. \& Oemler A. 1978, Astrophys.J., 219, 18-33.

4. Butcher H. \& Oemler A. 1984, Astrophys.J., 285, 426-38.

5. Dressler A, Oemler A., Butcher H. \& Gunn J.E. 1994a, Astrophys.J., 430, 107-20.

6. Dressler A, Oemler A., Sparks W.B. \& Lucas R.A. 1994b, Astrophys.J.Lett., 435, L23-6.

7. Couch W.J., Ellis R.S., Sharples R. \& Smail I. 1994, Astrophys.J., 430, 121-38.

8. Barger, A. J., Aragon-Salamanca, A., Ellis, R. S., Couch, W. J., Smail, I. and Sharples, R. M. 1995, Mon.Not.R.Astr.Soc., in press.

9. Griffiths R.E. et al 1994, Astrophys.J.Lett., 435, L19-22.

10. Schechter P. 1976, Astrophys.J., 203, 297-306.

11. Binggeli B., Tammann G.A. \& Sandage A. 1987, Astron.J., 94, 251-77.

12. Thomspon L.A.\& Gregory S.A. 1993, Astron.J., 106, 2197-212.

13. Kauffmann G. 1995, Mon.Not.R.astr.Soc., 274, 153-60.

14. Icke V. 1985, Astron.Astrophys., 144, 115-23.

15. Miller R.H. 1988, Comment. Astrophys., 13, 1-11.

16. Dressler A. \& Gunn J.E. 1983, Astrophys.J., 270, 7-19.

17. Evrard A.E. 1991, Mon.Not.R.astr.Soc., 248, 8p-10.

18. Byrd, G. and Valtonen-M. 1990, Astrophys.J., 350, 89-94.

19. Valluri M. 1993, Astrophys.J., 408, 57-70.

20. Oemler, A., Dressler, A. and Butcher, H. R. 1995, Astrophys.J., submitted.

21. Hernquist L. \& Katz N. 1989, Astrophys.J.Supp., 70, 419-46.

22. Tully R.B. \& Fisher J.R. 1977, Astro.Ap., 54, 661.

23. Faber S.M. \& Jackson R.E. 1976, Astrophys.J., 204, 668-83.

24. Moore B., Katz N. \& Lake G. 1995, Astrophys.J., in press.

25. White S.D.M. \& Rees M.J. 1978, Mon.Not.R.Astr.Soc., bf 183, 341-58.

26. van der Marel, R. P. 1991, Mon.Not.R.Astr.Soc., 253, 710-26.

27. Sandage, A. and Bedke, J. 1994, The Carnegie Atlas of Galaxies, Carnegie Institute of Washington.

28. Combes F., Dupraz C., Casoli F. \& Pagani L. 1988, Astron.Astrophys., 203, L9-12.

29. Kormendy, J. and Bender, R. 1995, in ESO/OHP Symposium on Dwarf Galaxies,, ed. G. Meylan and P. Prugniel, European Southern Observatory, in press.

30. Ferguson H.C. \& Binggeli B. 1994, Astronomy and Astrophysics Review, 6, 67-122.

31. Barnes J.E. \& Hernquist L. 1992, Nature, 360, 715-17.

32. Mirabel I.F., Dottori H. \& Lutz D. 1992, Astron.Astrophys., 256, L19-22.

33. Gudehus D.H. 1989, Astrophys.J., 340, 661-5.

34. Tyson J.A. \& Fischer P. 1995, Astrophys.J.Lett., in press.

35. Bahcall, J.N., Kirkhakos S. \& Schneider D.P. 1994, Astrophys.J. Lett., 435, L11-14.

36. Disney M.J. et al 1995, Nature, 376, 150-3.

37. French H.B. \& Gunn J.E. 1983, Astrophys.J., 269, 29-34.

38. Lake G., Katz N. \& Moore B. 1995, Astrophys.J., in press.

39. Toomre A. 1977, In The Evolution of Galaxies and Stellar Populations, ed. B.M. Tinsley \& R.B. Larson, p. 401-17. Yale University Observatory.

40. Ostriker J.P. \& Hausman M.A. 1977, Astrophys.J.Lett., 217, L125-9. 
This figure "article1a.jpg" is available in "jpg" format from: http://arXiv.org/ps/astro-ph/9510034v1 
This figure "article1b.jpg" is available in "jpg" format from: http://arXiv.org/ps/astro-ph/9510034v1 
This figure "article2.gif" is available in "gif" format from: http://arXiv.org/ps/astro-ph/9510034v1 
This figure "article3a.gif" is available in "gif" format from: http://arXiv.org/ps/astro-ph/9510034v1 
This figure "article3b.gif" is available in "gif" format from: http://arXiv.org/ps/astro-ph/9510034v1 
This figure "article3c.gif" is available in "gif" format from: http://arXiv.org/ps/astro-ph/9510034v1 
This figure "article3d.gif" is available in "gif" format from: http://arXiv.org/ps/astro-ph/9510034v1 
This figure "article3e.gif" is available in "gif" format from: http://arXiv.org/ps/astro-ph/9510034v1 
This figure "article3f.gif" is available in "gif" format from: http://arXiv.org/ps/astro-ph/9510034v1 
This figure "article4.gif" is available in "gif" format from: http://arXiv.org/ps/astro-ph/9510034v1 